\title{
An Advanced DV-hop Localization Algorithm in Wireless Sensor Network
}

\author{
Ming Jiang ${ }^{1}$, Yunfei $\mathrm{Li}^{2}$, Yuan $\mathrm{Ge}^{3}$, Wengen $\mathrm{Gao}^{4}$ and $\mathrm{Ke} \mathrm{Lou}^{5}$ \\ AnHui Key Laboratory of Detection Technology and Energy Saving Devices, AnHui \\ Polytechnic University, 241000,WuHu, AnHui ,China \\ ${ }^{1}$ Prof_Jiangming@163.com, 2nathonlee@163.com,3eyuan@ahpu.edu.cn, 4 \\ ahpuchina@ahpu.edu.cn, ${ }^{5}$ louke1979@163.com
}

\begin{abstract}
Position information is a fundamental element in WSN. As one of the range-free localization algorithm, the DV-Hop (Distance Vector-Hop) is widely applied. The DV-Hop localization algorithm employs the physical distance between the anchor nodes to estimate the average hop distance, instead of the actual transmission distance. The distance gap between the two kinds of distance undoubtedly leads to the error of the average hop distance as well as relatively high localization error. Aiming at solving the problem, the improved algorithm is proposed. The reason of error between the physical distance and actual transmission distance is analyzed in the paper, and summarized as the asymmetry of distance resulted from the nodes random distribution, as well as the deviation degree between the actual transmission path and the physical distance, and the distance compensation model is built based on the asymmetry and deviation degree to make the physical distance closer to the transmission distance. In the improved algorithm increases no extra hardware and complexity. Compared to the original DV-Hop algorithm, the simulation results show that the improved algorithm proposed in the paper increases the positioning accuracy of the unknown nodes.
\end{abstract}

Keyword: Wireless Sensor Network; Positioning Algorithm; nonuniformity; nonlinear degree; topological structure; $\mathrm{DV}$-Hop

\section{Introduction}

The wireless sensor network (WSN) has been applied in many fields, e.g. the distant medical system, military defense, smart transportation and environmental monitoring [1]. In these applications, the remote controller receives the data that carries the location where the data or events occurs, which will help the remote controller or human to monitor the goal objects, and the data is available information [2-4]. If the remote controller receives data without available location information, the data is not only meaningless, but also wastes the energy of nodes in transmission. Therefore, the position information of the nodes is of vital importance to the whole wireless sensor network and the applications.

Generally, according to the required information and hardware, the localization algorithms of the WSN are classified into two categories: Range-based and Range-free. The range-based localization algorithms include Time of Arrival (TOA), Received Signal Strength Indicator (RSSI), Time Difference of Arrival (TDOA), and Angel of Arrival (AOA) [5-7]. These algorithms all require extra hardware to get relevant information, $e . g$. the signal strength indicators are needed to measure the signal strength in RSSI. Though these algorithms have relatively high localization accuracy, they inevitably increase the cost when they are applied 
in large scale wireless sensor network. The range-based localization algorithms have no special requirements for extra information and hardware, so they are more suitable in large scale WSN with lower cost and energy consumptions, these algorithms mainly are: APTI, DV-Distance, MDS-Map, and DV-Hop (Distance Vector-Hop) [5-6].

In DV-Hop algorithm was proposed by D. Niculescu and B. Nath [7]. It is simple, robust and has good coverage quality, feasibility as well as facility. Therefore it was widely used in WSN. However, it was faced with two main disadvantages: relatively high power-consumption and inadequate localization accuracy [8-11].

The improved algorithm is proposed in order to improve the localization accuracy in the paper. Firstly, two categories errors are formed to depict the defect of the DV-Hop, and the mathematic model is built based on the analysis results, eventually, the verification of the mathematic model is simulated, and the conclusions are drawn.

\section{Related Work}

The original DV-Hop algorithm employs a distance vector exchange so that all anchor nodes get the distance and hops to the other anchor nodes, and all unknown nodes get hops to the nearest anchor node. Then the anchor nodes estimate the average distance per hop, which is a correction and transmitted to the entire networks. Finally, and the unknown nodes get their locations by multilateral measurement method. Generally, the original DV-Hop algorithm can be divided into three steps [11-13].

In the first step, the anchor nodes broadcast their information packages to all other nodes through neighbor nodes. The information packages contains anchor nodes' identifiers, location coordinates and hop count. The hop count is initially set zero, and increase by one when the package passes a node. A node will conclude the minimum hop count to the certain anchor nodes from all the information packages the node received [13-16]. If a node gets an information packages with different hop count to the same anchor node and the package with lowest hop count will be maintained. The maintained information package will be flooded outward to the entire network. The updates and further broadcasts will continue until all the nodes receive their shortest path. Eventually, every sensor node gets minimal hop count to a certain anchor node.

In the second step, anchor nodes get location coordinate and the minimal hop count to other anchor nodes, and average distance in one hop called average hop distance (AHD), and it is defined as:

$$
A H D_{\mathrm{i}}=\frac{\sum_{j \neq i}^{n} \sqrt{\left(x_{i}-x_{j}\right)^{2}+\left(y_{i}-y_{j}\right)^{2}}}{\sum_{j \neq i}^{n} h o p s_{i j}} \bullet
$$

In (1), $\left(x_{i}\right) y_{i},\left(x_{j}, y_{j}\right)$ are the respectively the coordinate of anchor node $i$ and $j$, and $n$ is the number of anchor nodes which are able to communicate with the anchor node

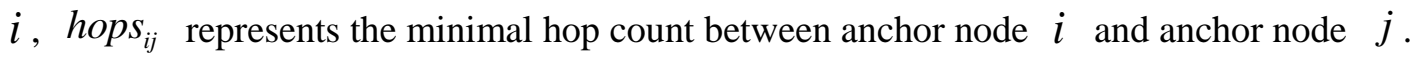

Each anchor node broadcasts its AHD to its neighbors. As the first step describes, the received sensor nodes flooded outward the AHD information. With accepting the AHD information from anchor node, the unknown node computes its distance to the anchor node as:

$$
d_{i j}=A H D_{j} \quad h o p
$$


In (2), hops $s_{i}$ is the hop count of the unknown node to the anchor node, and $A H D_{j}$ stands for the AHD information the unknown node accepts.

In the third step, each unknown node calculates its location coordinate with the equation set as:

$$
\left\{\begin{array}{c}
\sqrt{\left(x-x_{1}\right)^{2}+\left(y-y_{1}\right)^{2}}=d_{1} \\
\sqrt{\left(x-x_{2}\right)^{2}+\left(y-y_{2}\right)^{2}}=d_{2} \\
\cdots \\
\sqrt{\left(x-x_{n}\right)^{2}+\left(y-y_{n}\right)^{2}}=d_{n}
\end{array}\right.
$$

In (3), $(x, y)$ represents the location coordinate of the unknown nodes, and $\left(x_{i}, y_{i}\right)$ represents the anchor nodes location coordinates, and $d_{i}$ is the distance between the anchor nodes and the unknown nodes.

The equation set can be rewritten as: $A X=B$, and $A, B, X$ are as :

$$
\begin{aligned}
& A=\left[\begin{array}{ccc}
x_{1}-x_{n} & & y_{1}-y_{n} \\
x_{\mathrm{i}}-x_{n} & & y_{i}-y_{n} \\
& \ldots & \\
x_{n-1}-x_{n} & & y_{n-1}-y_{n}
\end{array}\right] . \\
& B=\left[\begin{array}{c}
d_{1}^{2}-d_{n}^{2}-x_{1}^{2}+x_{n}^{2}-y_{1}^{2}+y_{n}^{2} \\
d_{i}^{2}-d_{n}^{2}-x_{i}^{2}+x_{n}^{2}-y_{i}^{2}+y_{n}^{2} \\
\cdots \\
d_{n-1}^{2}-d_{n}^{2}-x_{n-1}^{2}+x_{n}^{2}-y_{n-1}^{2}+y_{n}^{2}
\end{array}\right] \text {. } \\
& X=\left[\begin{array}{l}
x \\
y
\end{array}\right] \text {. }
\end{aligned}
$$

The least square solution of $A X=B$ should be:

$$
X=\left(A^{T} A\right)^{-1} A^{T} B \text {. }
$$

\section{Improved DV-Hop Algorithm}

\subsection{Problem Description}

As mentioned above, the DV-Hop employs a distance vector exchange to calculate the distance between nodes, which results in distance error.

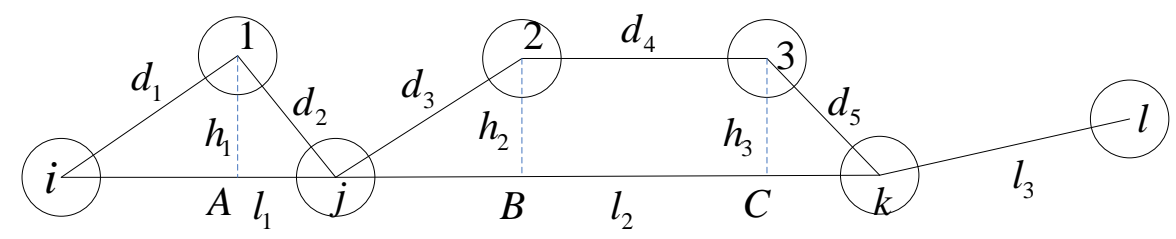

Figure 1. Part Network Topology of WSN 
As shown in the Figure 1, the anchor nodes $i, j, l, k$ and the unknown nodes 1, 2, 3 are deployed. According to (1), the AHD of the anchor node $i$ is calculated as:

$$
A H D_{i}=\frac{d_{i j}+d_{i k}+d_{i l}}{2+5+6} \approx \frac{3 l_{1}+2 l_{2}+l_{3}}{13}
$$

The anchor node $\mathrm{I}$ estimates the distance $d_{1}+d_{2}$ as:

$$
d_{\text {est }}=A H D_{i} \bullet H o p s=\frac{6 l_{1}+4_{2}+\mathcal{L}}{13}
$$

Based on the assumption $l_{2}>l_{1}>l_{3}>R$, the conclusion can be drawn as:

$$
d_{\text {error }}=\left|l_{1}-d_{\text {est }}\right|=\left|l_{1}-\frac{6 l_{1}+4 l_{2}+2 l}{13}\right| \beta=\left|\frac{7 l-4 l-2 l}{13}\right|>\left|\frac{7 l-6 l}{13}\right|>\frac{l}{13}
$$

Obviously, the distance $d_{1}+d_{2}$ is more than $l_{1}$, which leads to distance error. The unknown node 1 deviates from the line $i j$ which makes the $d_{1}+d_{2}>l_{1}$. If the unknown node 1 is deployed in the point A, namely there is no deviation degree. But the nonuniformity of the distance $l_{i A}$ and $l_{A j}$ exists, which means the distance between nodes varies randomly and will generates localization error.

\subsection{The Improved DV-Hop}

The above deduction shows that the DV-Hop leads to the relatively high localization error because of distance errors that are caused by the nonlinear degree of the node and the nonuniformity of distance between neighbors in the topological structure. Hence, the improved algorithm is proposed to estimate nonlinear degree and nonuniformity.

In Figure 1, the unknown node 1 deviates from the line $i j$ and the deviation degree is related directly to the height $h_{1}$. The more the deviation degree is, the greater the height is, therefore, the height $h_{l}$ is employed to represent the nonlinear degree. Moreover, the DV-Hop ignores the nonuniformity of distance, and causes positioning errors.

The actual transmission distance between the anchor node $i$ and the anchor node $j$ is as:

$$
\begin{aligned}
d_{1} & =\sqrt{\left(\alpha_{1} l_{1}\right)^{2}+\left(h_{1}\right)^{2}} . \\
d_{2} & =\sqrt{\left(\alpha_{2} l_{1}\right)^{2}+\left(h_{1}\right)^{2}} . \\
d & =d_{1}+d_{2} .
\end{aligned}
$$

In (11), the $\alpha_{1}, \alpha_{2}$ is defined as:

$$
\begin{aligned}
& \alpha_{1}=l_{i A} / l_{1} \\
& \alpha_{2}=l_{A j} / l_{1} \\
& \alpha_{l_{1}}=\left\{\alpha_{1}, \alpha_{2}\right\}
\end{aligned}
$$

As shown in Figure $1, l_{i A}$ is the distance between point $i$ and point $A$, and $l_{A j}$ is the distance between point $A$ and point $j$. In (15), $\alpha_{l_{1}}$ is called the nonuniformity of distance 
between the node 1 and its neighbors in the topological structure, and $h_{l}$ represents the deviation degree of the topological structure, namely, the nonlinear degree of the node 1 .

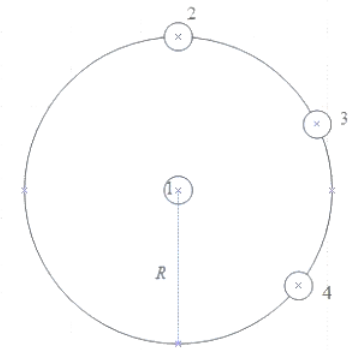

\section{Figure 2. Part of Ideal Network Topology in WSN}

The nonuniformity, representing the variance of distance between nodes, is zero under the assumption that the distance between neighbor nodes is equal. Radically, the nonuniformity represents the variance of density and distribution of nodes. In the ideal distribution of nodes, all nodes have the same distance to the neighbor nodes in communication radius, and all nodes are employed in uniform distribution and form an ideal network topological structure. Under these conditions, the positioning accuracy is only affected by the nonlinear degree.

In Figure 2, there are four nodes, and each pair neighbor nodes have the same distance, which is the communication radius R. Each pair neighbor nodes' distance is R. It is assumed that the neighbor nodes on the circle are at the margin of communication, and communicate with others through the center node 1 .

According to the rules, the amount of nodes that can be employed on the circle reaches the most at $2 \pi R / R=2 \pi$, and reaches the least at 1 . To the full extent, the positioning error only is caused by the nonlinear degree. The density of nodes is as:

$$
\begin{gathered}
\rho_{\text {m i }}=\frac{2}{\pi R^{2}} \\
\rho_{\max }=\frac{2}{R^{2}} \\
\frac{2}{\pi R^{2}} \leq \rho \leq \frac{2}{R^{2}}
\end{gathered}
$$

Ideally, when the density of nodes meets the demand of (19), the nodes density's variance does not have impact on the nonuniformity of distance between neighbors in the topological structure; otherwise, the nonuniformity will increase in exponential times. However, the actual distribution is more than that, and the nonuniformity of distance between neighbors is affected by distribution.

The nodes are employed in uniform distribution, and the density of nodes is $\rho$. There are $N$ nodes, and the probability $p_{\text {is }}$ that a node is employed in the certain region. So the $p\{x=m\}$ should be: 


$$
\begin{aligned}
P\{X=m\} & =\frac{m !}{m !(n-m) !} p^{m}(1-p)^{n-m} \\
& =\frac{n(n-1) \square(n-m+1)}{m !} \frac{\lambda^{m}}{m !}\left(1-\frac{\lambda}{n}\right)^{n-m} \\
& =\frac{\lambda^{m}}{m !}\left(1-\frac{1}{n}\right) \Gamma\left(1-\frac{m-1}{n}\right)\left(1-\frac{\lambda}{n}\right)^{n-m}
\end{aligned}
$$

Based on the Eq. (21) and (22),

$$
\begin{aligned}
& \lim _{n \rightarrow \infty}\left(1-\frac{1}{n}\right) \amalg\left(1-\frac{m-1}{n}\right)=1 \\
& \lim _{n \rightarrow \infty}\left(-1 \frac{\lambda}{n}{ }^{n-n}\right)=e^{-\lambda}
\end{aligned}
$$

The Eq. (20) can be rewritten as:

$$
P\{\mathrm{X}=m\}=\frac{\lambda^{m}}{m !} e^{-\lambda}
$$

In (23), $\lambda=n p$. So the probability that there are $X$ nodes in the region $S_{A}$ is $p\{x=m\}$,

$$
P\{\mathrm{X}=m\}=\frac{\left(\mathrm{S}_{A} \rho\right)^{m}}{m !} e^{-S_{A} \rho}
$$

From the Eq. (24), the conclusion that the nonuniformity is affected by the density of the nodes and changes in exponential times, can be drawn, namely, which can be expressed as:

$$
\alpha \propto e^{-\theta \rho}
$$

In Figure 1, the nonuniformity of the node 1,2 and 3 meets the demand as:

$$
\alpha=\sum_{i=1}^{\text {hops }} \alpha_{i}
$$

As mentioned above, the equation of $\alpha$ is:

$$
\alpha=\frac{l_{i}}{R \square h o p s} \cdot e^{-\rho \theta} \quad(\mathrm{i}=1,2)
$$

In (26), the density $\rho$ is expressed as:

$$
\rho=\left\{\begin{array}{cc}
0 & \rho<\rho_{\mathrm{min}} \\
1 & \rho_{\mathrm{m} \mathrm{ax}}<\rho<\rho \text { m } \\
\rho / \rho_{\mathrm{max}} & \rho>\rho \text { m a x }
\end{array}\right.
$$

Then, $\alpha_{i}$ can be calculated as:

$$
\alpha_{i}=P \square \frac{l}{R \square h o p s} \cdot \frac{(\rho \theta)^{k}}{k !} \quad(\mathrm{i}, k=1,2 \ldots, \text { hops })
$$

The nonlinear degree, representing the degree that actual transmission distance deviates from physical distance, is represented by the height, e.g. $h_{1}, h_{2}$ and $h_{3}$. In Figure 1 , the height $h_{1}, h_{2}$ and $h_{3}$ can be regarded as the distance between a point and a line in 
coordinates. The coordinates of $i, j$ respectively are $\left(x_{a}, y_{a}\right)$ and $\left(x_{b}, y_{b}\right)$, and the coordinates of the unknown nodes are $\left(x_{i}, y_{i}\right) i=1,2,3$.

Theorem.1. In the rectangular region $[a, b] \times[c, d]$, the coordinate of node $(X, Y)$ subjects to the uniformly distribution:

$$
f(x, y)=\left\{\begin{array}{c}
\frac{1}{(b-a)(d-c)} a \leq x \leq b \quad c \leq y \leq \\
0 \\
\text { else }
\end{array}\right.
$$

$X$ and $Y$ also follow the uniformly distribution, and they are mutually independent.

\section{Demonstration:}

1. Adequacy:

$X \sim U[a, b], \mathrm{Y} \sim U[c, d]$, and the density functions of $X$ and $\mathrm{Y}$ are as:

$$
\begin{gathered}
f_{X}(x)=\left\{\begin{array}{cc}
\frac{1}{b-a} & a \leq x \leq b \\
0 & \text { else }
\end{array}\right. \\
f_{Y}(y)=\left\{\begin{array}{cc}
\frac{1}{d-c} & c \leq x \leq d \\
0 & \text { else }
\end{array}\right.
\end{gathered}
$$

Because $X$ and $\mathrm{Y}$ are mutually independent, so the joint density function of $(X, Y)$ is as:

$$
\begin{aligned}
f(x, y) & =f_{X}(x) \bullet f_{Y}(y) \\
& =\left\{\begin{array}{cc}
\frac{1}{(b-a)(d-c)} & a \leq x \leq b, c \leq y \leq d \\
0 & \text { else }
\end{array}\right.
\end{aligned}
$$

So $(X, Y)$ subjects to the uniform distribution in $[a, b] \times[c, d]$.

\section{Necessity}

$(X, Y)$ subjects to the uniform distribution in $[a, b] \times[c, d]$, so the marginal density functions of $X$ and $\mathrm{Y}$ are respectively as (31) and (32). Therefore,

$$
f(x, y)=f_{X}(x) \bullet f_{Y}(y)
$$

So $X$ and $\mathrm{Y}$ are mutually independent, and $X \sim U[a, b], \mathrm{Y} \sim U[c, d]$.

Combined with above, the height $h_{1}$ can be calculated in three different situations.

(1) $x_{a} \neq x_{b}, y_{a} \neq y_{b}$

The equation of line ${ }_{\text {ij }}$ is as:

$$
\left(x_{a}-x_{b}\right) y-\left(y_{a}-y_{b}\right) x+y_{a} x_{b}-y_{b} x_{a}=0
$$

The height $h_{1}$ can be expressed as: 


$$
\begin{aligned}
h_{1}=d & =\frac{\left|\left(x_{a}-x_{b}\right) y_{1}-\left(y_{a}-y_{b}\right) x_{1}+y_{a} x_{b}-y_{b} x_{a}\right|}{\sqrt{\left(x_{a}-x_{b}\right)^{2}+\left(y_{a}-y_{b}\right)^{2}}} \\
& =\frac{\left|A x_{1}+B y_{1}+C\right|}{l_{1}}
\end{aligned}
$$

So the distribution function is:

$$
F_{H}(h)=\iint_{H<h} f(x, y) d x d y
$$

The probability density function is:

$$
\begin{aligned}
f_{H}(h) & =\frac{1}{D(b-a)(d-c)} & & a \leq x \leq b<1 \leq \\
& =\frac{h d-C}{(b-a)(d-c)}-\frac{A}{2 B} & & a \leq x \leq b, c \leq y \leq d
\end{aligned}
$$

In (38), $D=\frac{B}{l_{1}}$, and the height subjects to uniformly distribution.

(2) $x_{a}=x_{b}$

The height $h_{1}$ is expressed as:

$$
h_{1}=\left|x-x_{a}\right|
$$

So the distribution function is:

$$
\begin{aligned}
F_{H}(h) & =\iint_{|x-x|<h} f(x, y) d x \\
& = \begin{cases}\frac{h+x_{a}}{(b-a)(d-c)} & a<x<x_{a}+h \\
\frac{b+h-x_{a}}{(b-a)(d-c)} & x_{a}-h<x<b\end{cases}
\end{aligned}
$$

The probability density function is:

$$
f_{H}(h)=\frac{1}{(b-a)(d-c)} \quad a \leq x \leq b, c \leq y \leq d
$$

(3) $y_{a}=y_{b}$

Evidenced by the same token, the probability density function is:

$$
f_{H}(h)=\frac{1}{(b-a)(d-c)} \quad a \leq x \leq b, c \leq y \leq d
$$

In Figure 3, the node $\mathrm{A}$ and node $\mathrm{C}$ cannot directly communicate with each other, and the information is transmitted by the node $\mathrm{B}$. The node $\mathrm{B}$ must be in the overlapping area of two communication radius. In Fig.3, the height $h$ maximizes when the node $\mathrm{B}$ is at the location as shown in Figure 3. If the distance between the node A and B generally declines, the overlapping area expands, which make the height increase. The distance between the node $\mathrm{A}$ and $\mathrm{B}$ reaches the marginal value at $\mathrm{R}$, and the height is $\sqrt{3} R / 2$. If the distance between the 
node A and B generally increases the distance reaches the largest value at $2 \mathrm{R}$ and the height is 0 . If the distance continues to grow, there is no overlapping area, and one more relay node will be needed.

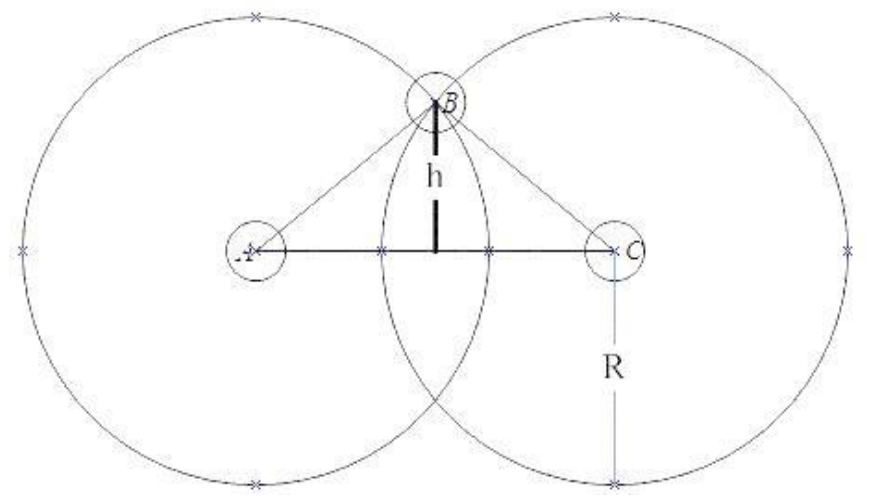

Figure 3. The Height in Part of Network Topology in WSN

Combined with the described above, the height can be calculated as:

$$
h_{i}=Q \square \operatorname{rand}(0,1) \square \frac{\sqrt{3}}{2} R \llbracket e^{(\rho-1) \varphi}
$$

In (44),

$$
\rho=\left\{\begin{array}{cc}
0 & \rho<\frac{3}{\pi R^{2}} \\
1 & \frac{3}{\pi R^{2}}<\rho<\rho_{\text {max }} \\
\frac{\rho_{\max }}{\rho} & \rho_{\text {max }}<\rho
\end{array}\right.
$$

rand $(0,1)$ represents the uniform distribution in $[0,1]$.

So, the exact distance can be estimated as:

$$
d=\sqrt{\left(\alpha_{i} l_{j}\right)^{2}+\left(h_{i}-h_{i-1}\right)^{2}} \quad(i=1,2,3, \ldots, \text { hops })
$$

In the second step of DV-Hop, the exact distance is estimated with (45).

\section{Simulation and Conclusion}

To evaluate the feasibility and validity of the improved algorithms proposed in the paper, simulations of comparison between the improved algorithm and the original DV-Hop algorithm are presented. The performance of the two algorithms both are simulated 200 times.

One hundred nodes, including 90 unknown nodes and 10 anchor nodes, are deployed in the area with both length and width are 100 as is shown in the Figure 4, where the red pots represent the anchor nodes and the blue pot is unknown nodes. Average localization error, under different communication radius and anchor ratios, was selected as the criterion to evaluate the performance of the algorithms.

$$
\mathrm{Er}=\frac{\sqrt{\left(x-x_{e s t}\right)^{2}+\left(y-y_{\text {esst }}\right)^{2}}}{R} .
$$




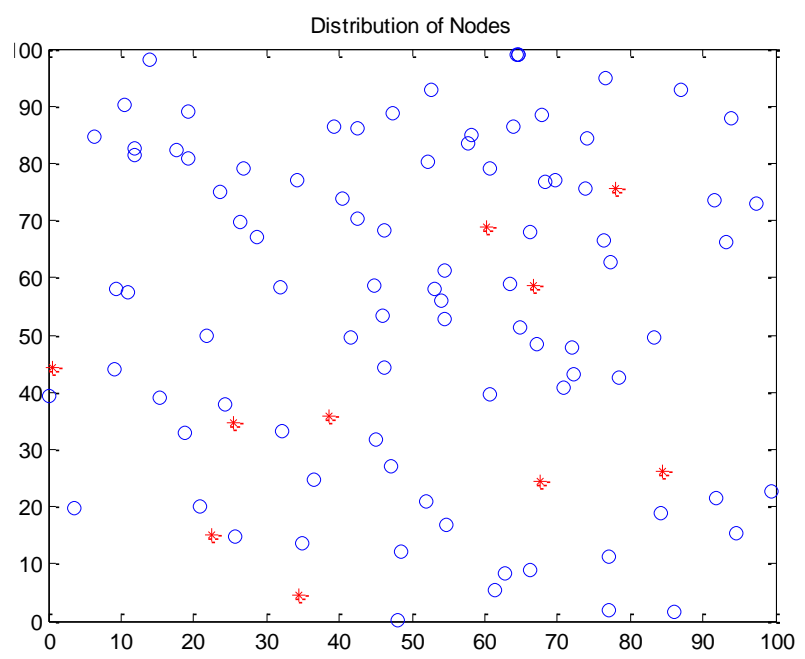

Figure 4. Nodes of Random Distribution in $100 * 100$

In Figure 5, the localization accuracy of the DV-Hop and the improved DV-Hop is simulated in different anchor ratios and communication radius with the node sum of 100 , and Figure 5 is the front view.

The communication radius ranges from 20 to 50, and the anchor ratio ranges from $6 \%-40 \%$. The surfaces of two algorithms are both in downward trend, which means that the average localization errors both decline. The improved DV-Hop has better performance in localization error, and has much sharper trend than the DV-Hop. The surfaces of the two algorithms lean to low localization error with the increase of the anchor ratio and communication radius.

With the augment of the communication radius, the nonuniformity of topological structure fluctuates in a relatively small range, and the nonuniformity $\alpha_{i}$ reflects the fluctuation range. Meanwhile, the variance of the communication radius also affects the nonlinear degree. The increase of the communication radius lowers the relative density $\rho_{\max } / \rho$, and the nonlinear degree is under the joint impact of the communication and the relative density. So the exact distance can be estimated in better condition, which will decrease the localization error.

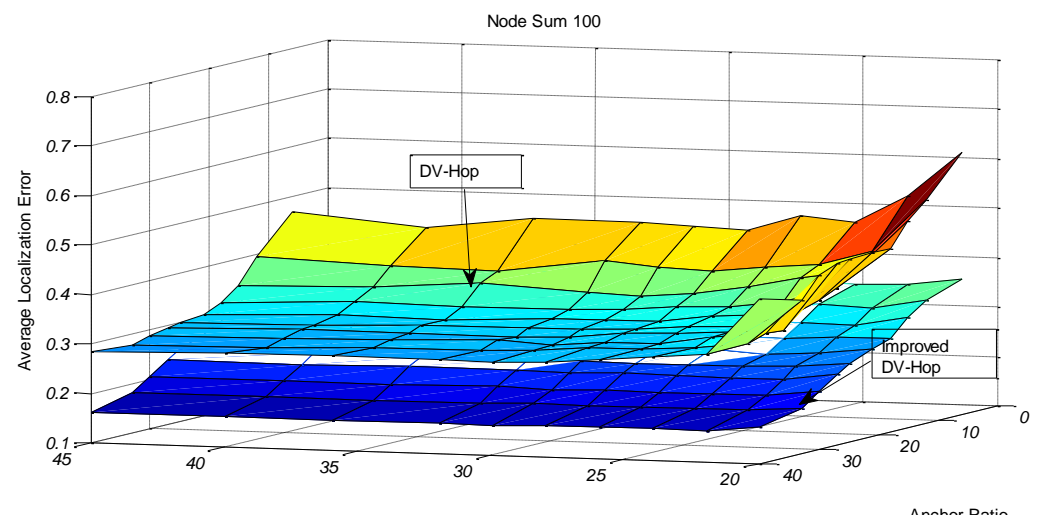

Communication Radius

Figure 5. Front View of the Average Localization in Different Anchor Ratios and Communication Radius 
In Figure 6, the localization error declines with the increase of the anchor node ratio. The anchor node estimates the $A H D$, and spread it to the entire network. The more the anchor nodes are, the more precise $A H D$ is when the anchor nodes employ the method of Eq. (42), and the better performance in localization error is achieved.

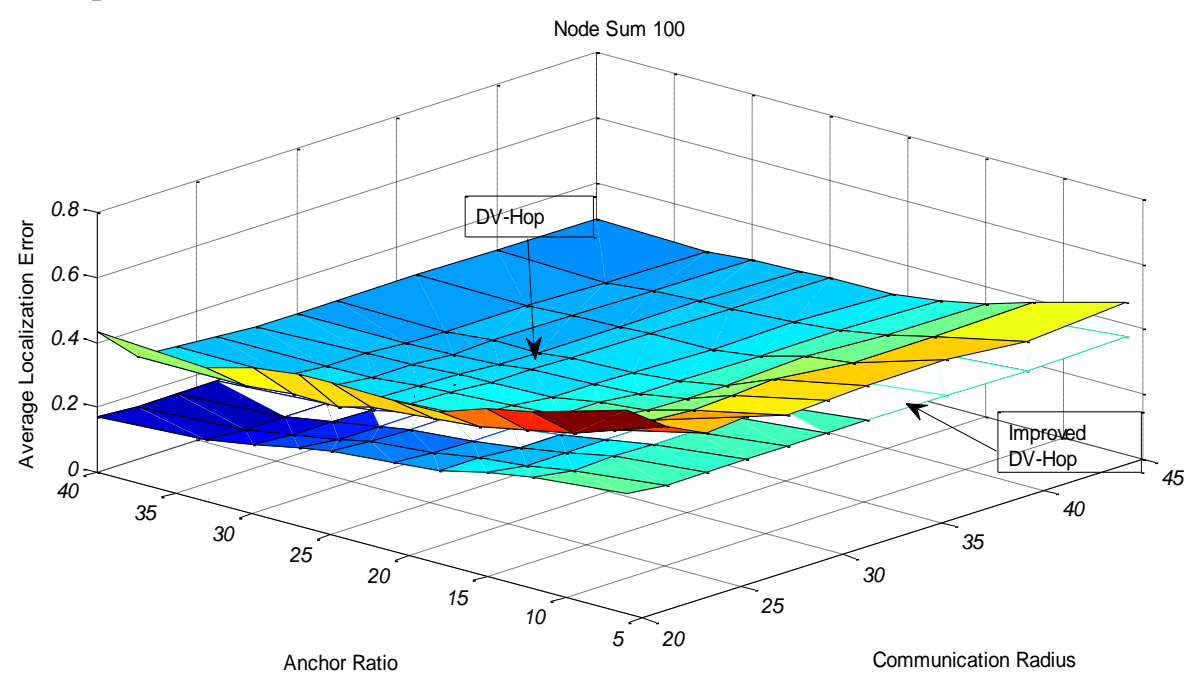

Figure 6. Top View of the Average Localization in Different Anchor Ratios and Communication Radius

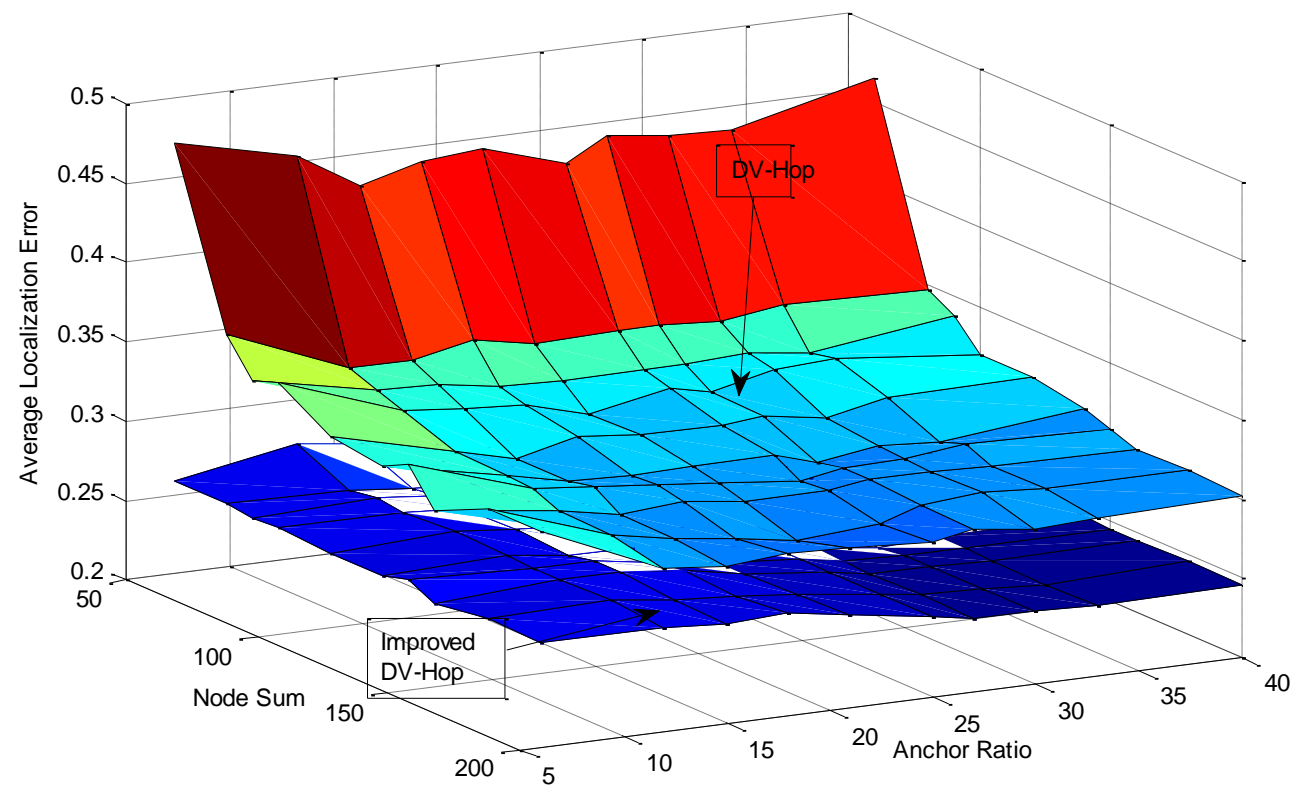

Figure 7. Side View of the Average Localization in Different Anchor Ratios and Node Sum

Figure 7 and Figure 8 shows the simulations with different node sum and different communication radius. The node sum ranges from 60 to 200, and the anchor ratio ranges from $6 \%-40 \%$, and the communication radius are 30 .

With the increase of the node sum and the anchor ratio, the average localization errors of two algorithms gradually decline, and the improved algorithm has better performance than the 
DV-Hop. When the node sum and anchor ratio is large enough, the increase of the node sum and the anchor ratio lead to augment of the hops and the density of nodes, which affects the nonuniformity and the nonlinear degree of the topological structure to vary slightly, and can improve little localization accuracy.

It is the amount of anchor node that affects the value of $h_{i}$ and $\alpha_{i}$, though the density of nodes also has impact on the nonuniformity and nonlinear degree. So at the same anchor ratio, the gap of two algorithms falls in limited range with the increase of the node sum, and at the same node sum, the gap of the two algorithms falls in larger range with the increase of the anchor ratios.

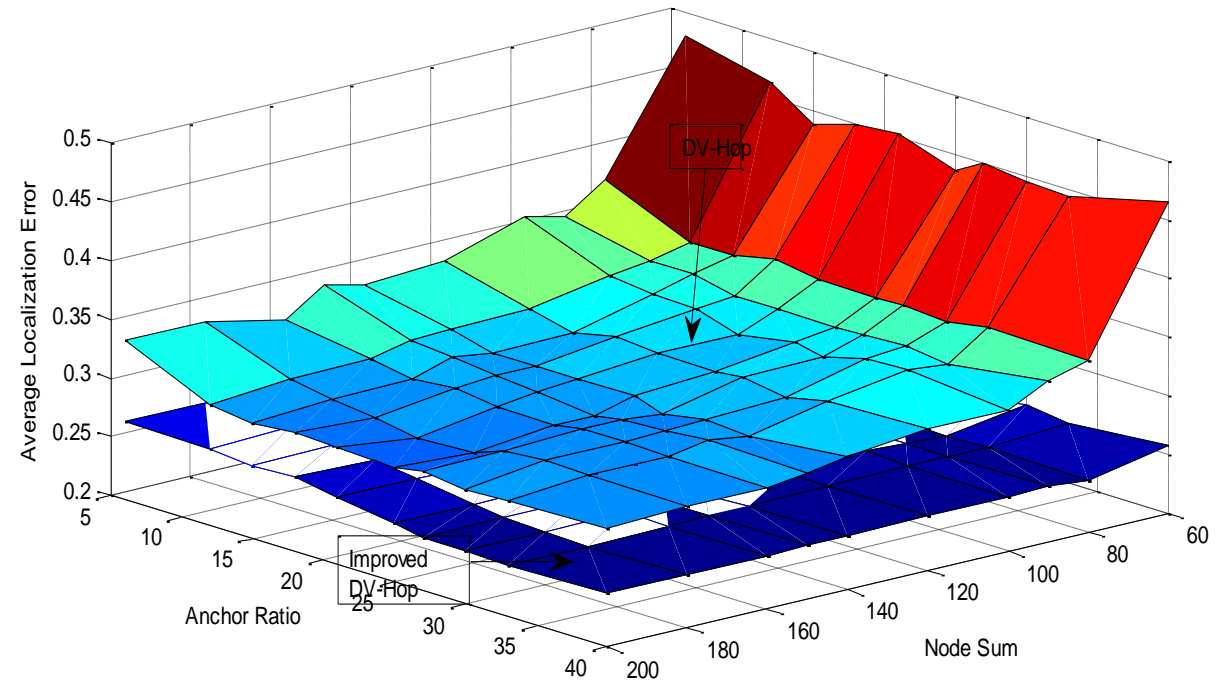

Figure 8. Front View of the Average Localization in Different Anchor Ratios and Node Sum

Figures 9-11 shows the simulation with different node sum and different communication radius. The node sum ranges from 60 to 200, and the communication radius ranges from 22 to 45 , and the anchor ratios are $20 \%$.

Basically, the changing trends of the two algorithms are the same with the increase of the communication radius and node sum. The error peak in the figures is resulted from the short communication radius and scant node sum, however, the improved DV-Hop can efficiently estimate the distance between the anchor nodes and the unknown nodes, and lower the localization error.

When the node sum reaches 50 and the communication radius is beyond 30 , the performance of the localization error is closest. Because according to (28), (40), when the low node sum is relatively low, it has more impact on the density of nodes and the topological structure than the communication radius has, which means that the nonuniformity and the nonlinear degree fluctuates relatively drastically, and the improved algorithm has limited promotion in localization error. The gap of localization errors in the transverse view, compared with the one in longitudinal view, changes more drastically. 


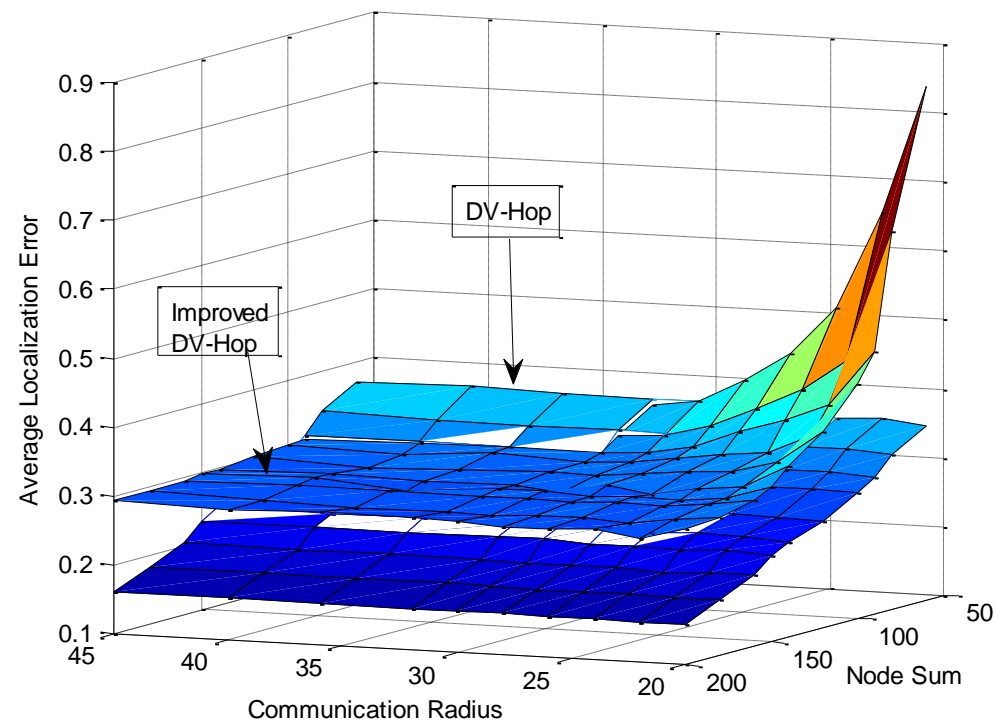

Figure 9. Front View of the Average Localization in Different Communication Radius and Node Sum

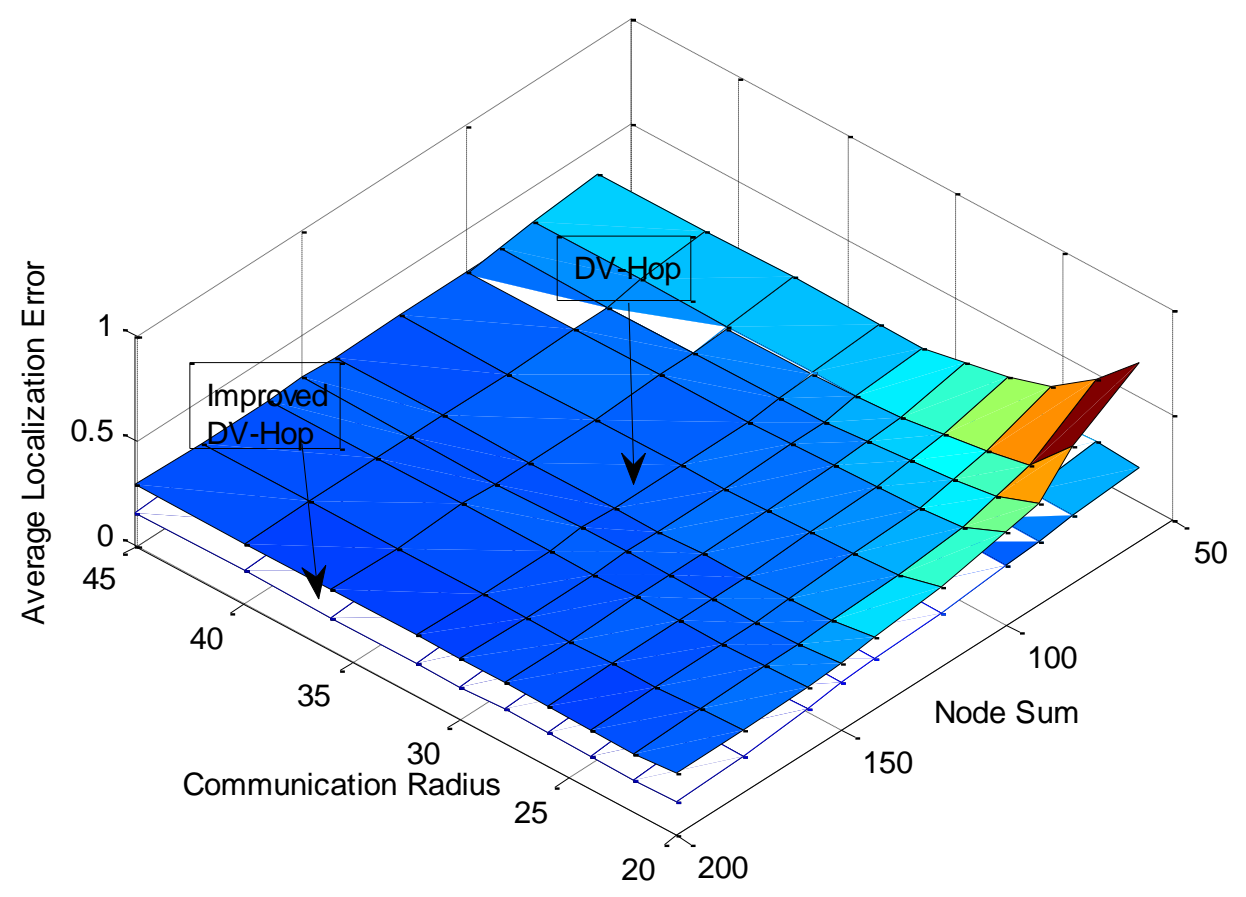

Figure 10. Top View of the Average Localization in Different Communication Radius and Node Sum 


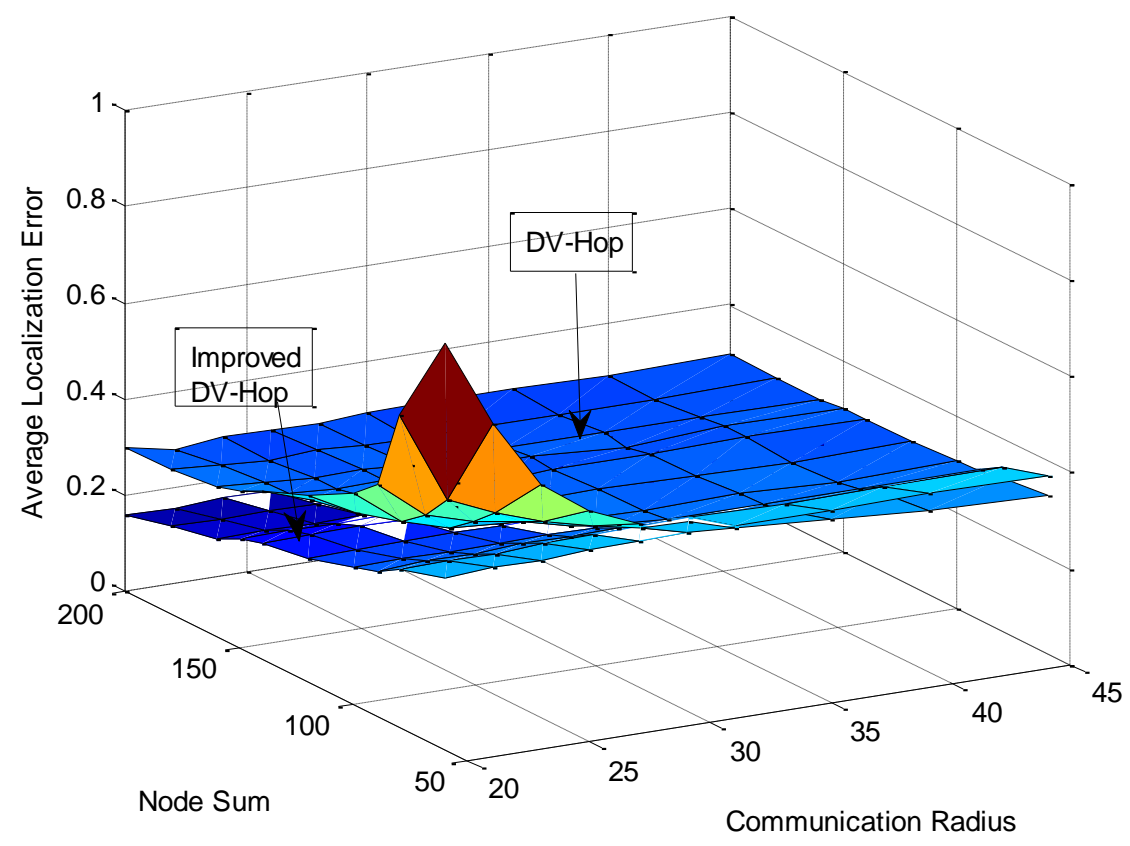

Figure 11. Rear View of the Average Localization in Different Communication Radius and Node Sum

The Figure 13 and Figure 14 shows the simulation of the average surplus energy of nodes. The transmission energy consumption model of nodes is as:

$$
E_{t}(i)=\left\{\begin{array}{cc}
\left(e_{t}+e_{d} \varepsilon_{f s} r^{2}\right) B & d<d_{0} \\
\left(e_{t}+e_{d} \varepsilon_{a m p} r^{4}\right) B & d \geq d_{0}
\end{array}\right.
$$

In (47), $E_{t}(i)$ represents the energy that the node $i$ consumes to send data, and $e_{t}$ is the energy that a node consumes to send a bit of data, and $e_{d}$ is rate of energy loss in the wireless transmission. $n_{\text {is }}$ the channel path loss exponent, and it depends on the RF environment. $r$ is the transmission distance, and $B$ is the wireless signal bit rate. ${ }^{\varepsilon_{f s}}$ is the acceptable bit error rate in the free transmission space, and $\varepsilon_{a m p}$ is the acceptable bit error rate in the multi-channel attenuation transmission. $d_{0}$ is the distance threshold, and is defined as:

$$
d_{0}=\sqrt{\frac{\varepsilon_{f s}}{\varepsilon_{a m p}}}
$$

The receiving energy consumption model of nodes is as:

$$
E_{t}(i)=e_{D A}+e_{r} B
$$

In (49), $E_{r}(i)$ represents the energy that the node $i$ consumes to receive data, and $e_{r}$ is the energy that a node consumes to receive a bit of data. $e_{D A}$ is the energy that the node $i$ consumes to merge data. 


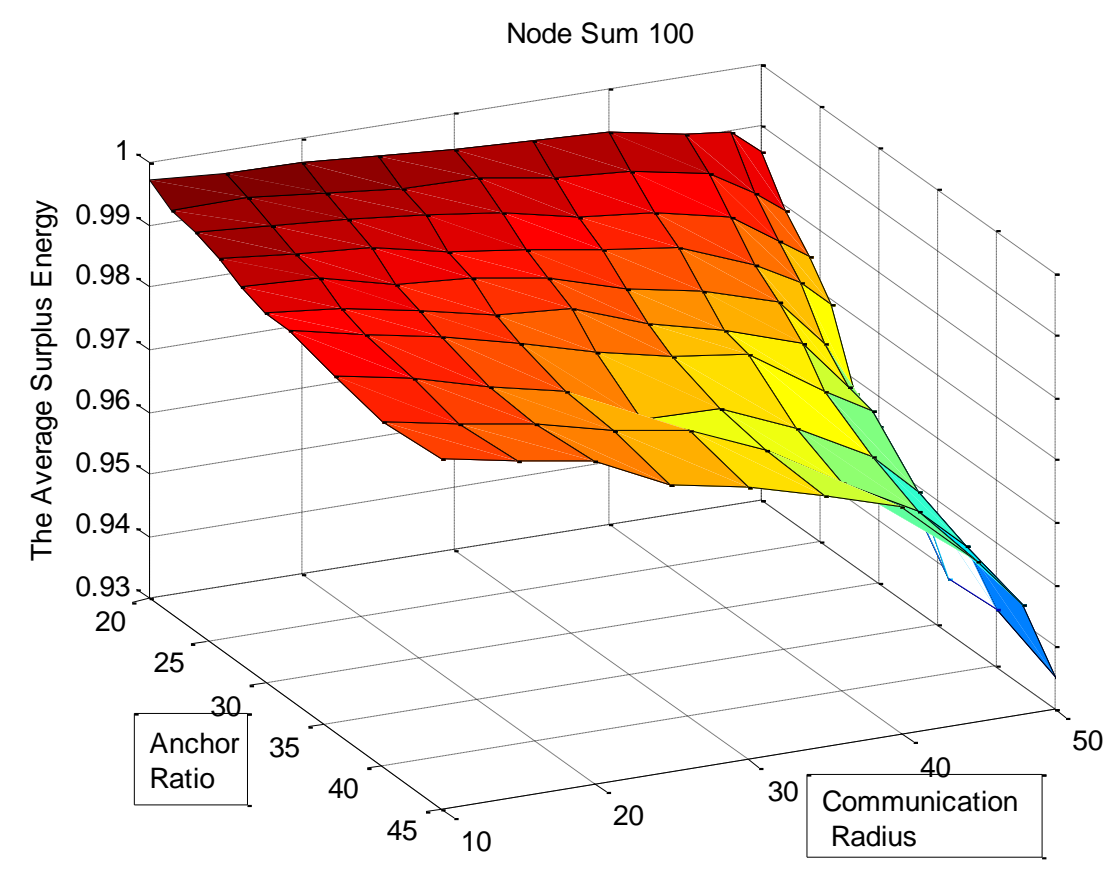

Figure 12. Side View of the Average Surplus Energy of Nodes in Different Anchor Ratios and Communication Radius

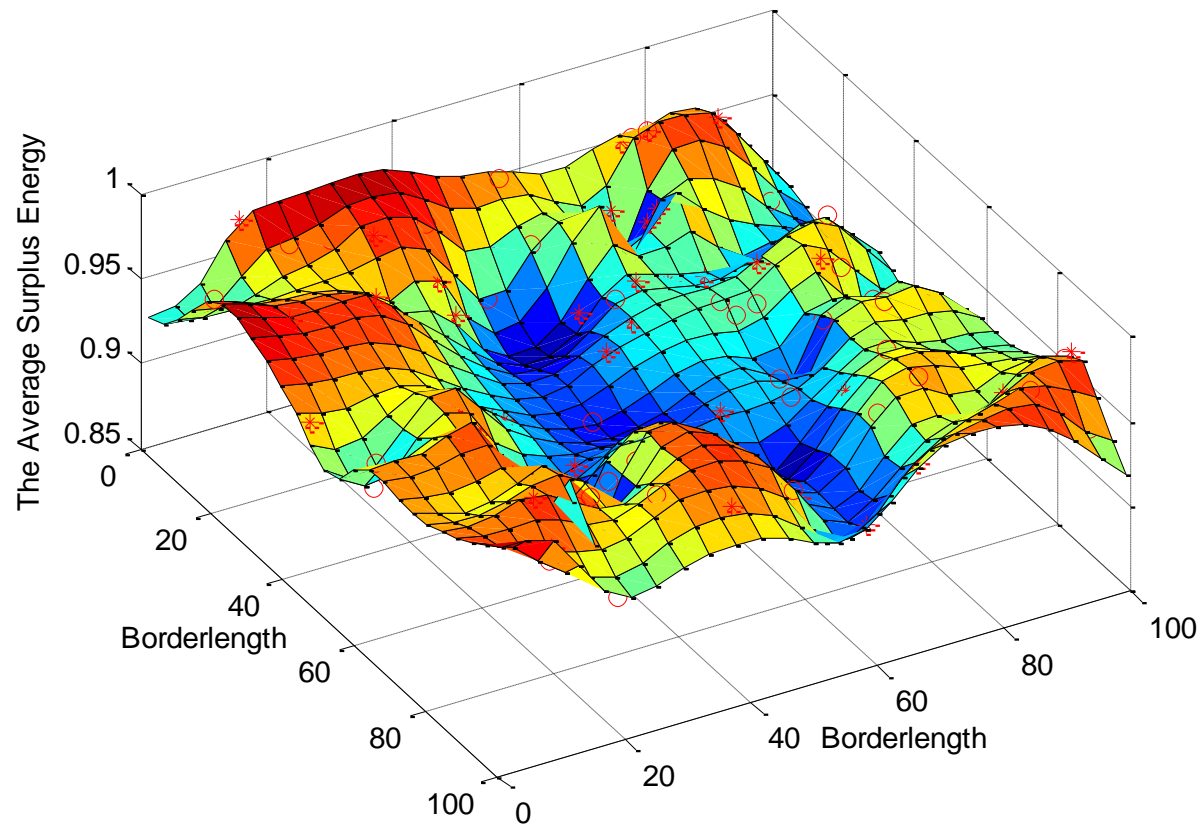

Figure 13. Side View of the Average Surplus Energy of Nodes in Different Coordinates 


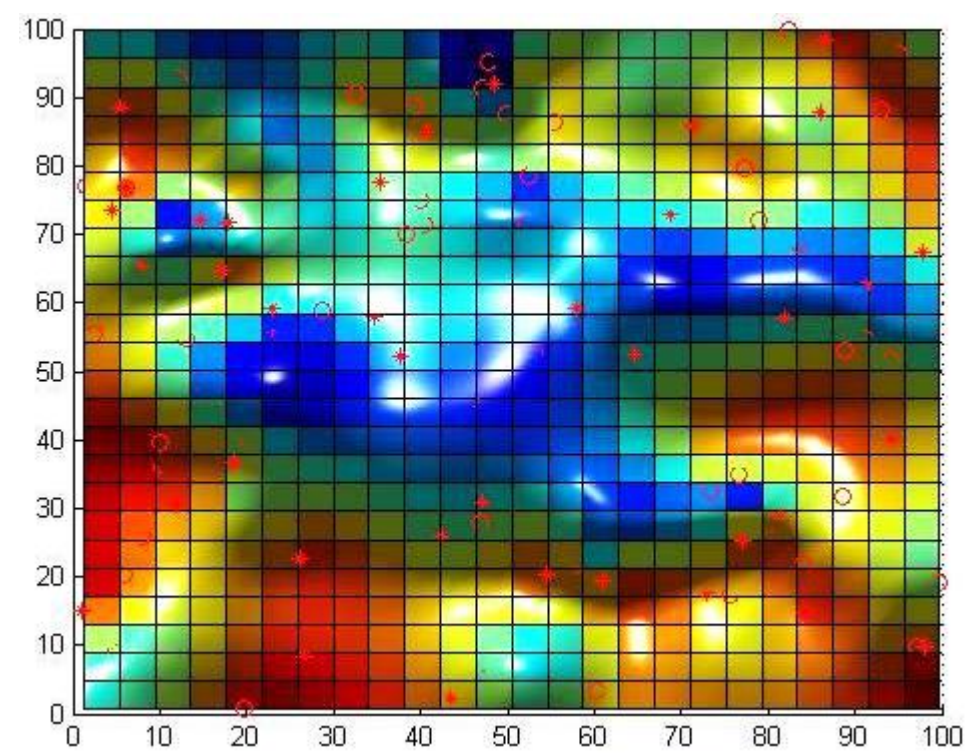

Figure 14. Top View of the Average Surplus Energy of Nodes in Different Coordinates

In Figure 12, the simulation result shows that the average surplus energy of nodes in different anchor ratios and communication radius. The average surplus energy of nodes gradually declines with the augment of the anchor ratio and communication radius. The enlargement of communication radius leads to the increase of the power of nodes, which results in the more energy consumption. Meanwhile, the increase of the anchor ratios makes the transmission of data much more, undoubtedly, which consumes more energy of nodes.

In Figure 13 and Figure 14, the simulation results show the surplus energy of nodes with different coordinates, and the vertical axis represents the surplus energy of nodes. The small red circle represents the anchor nodes, and the small red stars represent the unknown nodes.

The main energy consumption comes from the retransmission of data to the neighbor nodes. In DV-Hop, the distance between neighbor nodes is estimated by the AHD, which is not accurate enough to calculate the consumption energy. The nodes cannot estimate their energy precisely.

Although the improved DV-Hop transfers a little more data in localization, it provides more accurate distance between neighbor nodes, which makes the energy consumption quite low, and the two algorithms are so closed that the two algorithms compose a surface in Figure 13 and Figure 14.

In Figure 13, the nodes located in the margin maintain more energy, because these nodes do not undertake much transmission tasks, and the energy consumption is much lower.

In Figure 14, the areas with more nodes, the surplus energy of nodes are more. In the blue area, where not much nodes are not deployed, the surplus energy of nodes are lower than other nodes. Because, the nodes in the blue area are relatively more distant from other nodes, and it takes more power to retransmit data.

\subsection{Conclusion}

With the analysis of the DV-Hop, the defect of the DV-Hop is presented. Aiming at the reasons leading to the defect, the nonuniformity and nonlinear degree is proposed and mathematic proof is presented, and the model of nonuniformity and nonlinear degree is built. 
Based on the analysis and the simulation results, different factors have different impacts on the localization error, and the anchor ratio affects the most, and the communication comes second, then the node sum under the condition of the same interest area. The improved DV-Hop has better performance than the DV-Hop in localization in different conditions, and the improved DV-Hop is basically the same with the DV-Hop in energy consumption, which both testify the validity and feasibility of the model and the improved DV-Hop.

\section{Acknowledgement}

The paper was supported by Natural Science Foundation of China $(61271377 ; 61203034)$, Science and technology project of Anhui Province (1206c0805006), and Natural Science Fund Project of Colleges and universities in Anhui Province (KJ2012A035).

\section{References}

[1] J. Yick, B. Mukherjee and D. Ghosal, "Wireless sensor network survey [J]", Computer Networks, vol. 52, no. 2, (2008), pp. 2292-2230.

[2] D. Niculescu, "Positioning in Ad Hoc Sensor Networks [C]", IEEE Network, vol. 18, no. 4, (2004), pp. 24-29.

[3] K. Chen, Z. H. Wang, M. Lin and M. Yu, "An Improved DV-Hop Localization Algorithm for Wireless Sensor Networks [C]", IET International Conference on Wireless Sensor Network, (2010), pp. 255-259.

[4] G. Q. Gao and L. Lei, "An improved node localization algorithm based on DV-HOP in WSN [C]", Advanced Computer Control (ICACC), 2010 2nd International Conference on, vol. 4, (2010) March, pp. 27-29, pp. 321, 324.

[5] D. Niculescu and B. Nath, "Ad hoc positioning system (APS) using AOA [C]", Proceedings of the 22nd nnual Joint Conference of the IEEE Computer and Communications Societies, March 30-April 3, (2003), Rutgers University, Piscataway, pp. 1734-1743.

[6] Y. Zhang, K. Wang, S. Yuan, H. Yang, Z. Chen and L. Ge, "Research of WSN Node Localization Algorithm Based on Weighted DV-HOP [C]", 24th Chinese Control and Decision Conference, (2012), pp. 3826-3829.

[7] D. Niculescu and B. Nath, "DV based Positioning in Ad Hoc Networks [J]", Journal of Telecommunication Systems, vol. 22, no. 14, (2003), pp. 267-280.

[8] X. Yi, Y. Liu, L. Deng and Y. He, "An Improved DV-Hop Positioning Algorithm with Modified Distance Error for Wireless Sensor Network [C]”, The 2nd International Symposium on Knowledge Acquisition and Modeling, vol. 2, (2009), pp. 216-218.

[9] R.-J. Wang, B. Zhang, Y. Shen, Z.-G. Qin, J.-h. Wang, "PHDV-Hop: A More Accurate DV-Hop Positioning Algorithm in WSN [J]", JDCTA: International Journal of Digital Content Technology and its Applications, vol. 6, no. 13, (2012), pp. 89-97.

[10] Y. Dai, J. Wang and C. Zhang, "Improvement of DV-Hop Localization Algorithms for Wireless Sensor Networks [C]", Wireless Communications Networking and Mobile Computing (WiCOM), 2010 6th International Conference on , pp. 1, 4, (2010), September 23-25.

[11] X. D. Yu, M. Y. Zhang, M. Q. Zhu, K. H. Xu and Q. C. Xiang, "Improved DV-Hop Algorithm for Enhancing Localization Accuracy in WSN [J]', Applied Mechanics and Materials, vol. 543 - 547, (2014), pp. 3256.

[12] S. Kumar and D. K. Lobiyal, "An Advanced DV-Hop Localization Algorithm for Wireless Sensor Networks [J]”, Wireless Personal Communications, vol. 71, (2013) July, pp. 1365-1385.

[13] S. Tomic and I. Mezei, "Improved DV-Hop Localization Algorithm for Wireless Sensor Networks [C]", IEEE 10th Jubilee International Symposium on Intelligent Systems and Informatics, September 20-22, (2012), pp. 389-394.

[14] J. Zhang, "Node Localization Algorithm Based on DV-HOP in Wireless Sensor Networks [J]", (2013), Applied Mechanics and Materials, vol. 440, p. 289.

[15] Z. Huang and S. Li, "The Study on WSN Node Positioning Algorithm Improvement [J]", 2012 International Conference on Computer Science and Service System, (2012), pp. 1248-1249.

[16] Q. Qian, X. Shen, and H. Chen, "An Improved Node Localization Algorithm Based on DV-Hop for Wireless Sensor Network [J]”, Comput. Sci. Inf. Syst, (2011), pp. 953-972. 


\section{Authors}

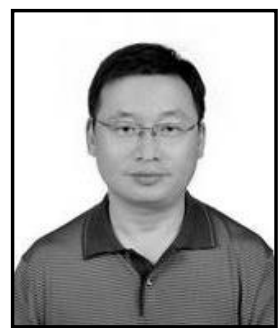

Ming Jiang, He was born in 1965 in Wuhu, Anhui, China, and received his master degree at Shanghai University. He is the vice director of AnHui Key Laboratory of Detection Technology and Energy Saving Devices, and a professor in Cyber Physical System.

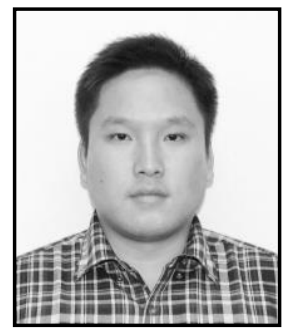

Yunfei Li, He was born in 1989 in Anqing, Anhui, China, and studys for his master degree at Anhui Polytechnic University. His research area is wireless sensor network localization. 